\title{
Sind die Geheilten aber auch gesund?
}

$\mathrm{M}$ ittlerweile überleben jedes Jahr ca. 1.450 der jährlich etwa 1.800 neu an Krebs erkrankenden Kinder und Jugendlichen in Deutschland - das sind über $80 \%$ [Creutzig et al. Dtsch. Ärztebl. 2003;100:A842-52; Kinderkrebsregister Jahresbericht 2009]. Viele leben anschließend ohne Einschränkungen mitten unter uns. Jedoch nicht alle...

Die standardisierte Mortalitätsrate (SMR) der langzeitüberlebenden jungen Erwachsenen fünf Jahre nach Diagnosestellung ist im Vergleich zur Normalbevölkerung dreifach erhöht. Dabei ist die Mortalität durch Rezidive und Zweitmalignome nicht berücksichtigt [Möller et al. J Clin Oncol. 2001;19(13):3173-81].

$\mathrm{Zu}$ den bedeutsamen Spätfolgen nach einer Krebserkrankung im Kindesalter gehören:

- Kardiomyopathien durch Anthrazykline,

- Hörverluste durch Platinderivate,

- Einschränkungen der Nierenfunktion durch Ifosfamid,

- Störungen endokriner Funktionen mit Minderwuchs,

- Hypothyreose und Infertilität durch Strahlentherapie und Alkylanzien,

- Neuropsychologische und kognitive Leistungsstörungen durch Schädelbestrahlung in Kombination mit Chemotherapie und

- Zweitmalignome durch Strahlentherapie und Topoisomerase-II-Inhibitoren.

In den USA leben derzeit mehr als zehn Millionen Krebsüberlebende, von denen 250.000 im Kindesund Jugendalter erkrankt waren [Meadows AT. J Clin Oncol. 2006;24(32):5160-5]. In Deutschland gibt es fast 30.000 ehemals krebskranke Kinder und Jugendliche und die Gesamtzahl der Überlebenden steigt jährlich [Kinderkrebsregister Jahresbericht 2009; Wallace WH. BMJ. 2001;323(7307):271-4].

\section{Spätfolgen werden in Studien untersucht}

Um Kollektive ehemaliger Patienten zu erforschen wurden in den USA und in Großbritannien große retrospektive Studien initiiert (USA: Childhood Cancer Survivor Study - CCSS; Großbritannien: British Childhood Cancer Survivor Study - BCCSS). In Deutschland startete 1998 das „Late Effects Surveillance System“ (LESS), in dem die Überlebenden bundesweit prospektiv auf Spätfolgen untersucht werden [Peeters J et al. Klin Padiatr. 2009;221(3):156-61].

Die Nachsorge der Kinder und Jugendlichen wird entsprechend ihres Risikoprofils (Erkrankung, Be- handlung, Alter, Prädisposition, u.a. Faktoren) geplant und durchgeführt. Untersuchungsempfehlungen für die einzelnen Patientengruppen sind unter den Nachsorge-Leitlinien der AWMF (www. awmf.org/leitlinien/detail/ll/025-003.html), auf der Homepage der GPOH (www.kinderkrebsinfo. de) oder unter www.less-studie.de zu finden. Das ist besonders für die niedergelassenen Pädiater wichtig, die die Patienten neben dem erstbehandelnden kinderonkologischen Zentrum weiterbetreuen.

\section{Spezialisten und niedergelassene Kollegen sind gemeinsam gefordert}

Ziel der Nachsorge ist es, Spätfolgen möglichst früh zu entdecken und dann zu behandeln. Dementsprechend benötigen wir Spezialisten aus verschiedenen Fachgebieten, die ehemals krebskranke Kinder, Jugendliche und junge Erwachsene in einer Nachsorgesprechstunde bzw. -klinik zusammen mit den niedergelassenen Kollegen betreuen. Solche Einrichtungen entstehen derzeit an den großen kinderonkologischen Zentren in Deutschland.

Viele ehemals krebskranke Kinder und Jugendliche erreichen das Erwachsenenalter, finden dann aber keinen auf die Nachsorge spezialisierten Arzt. Ein Ansatz, diese Versorgungslücke zu schließen, wäre die Anbindung ehemals krebskranker Kinder und Jugendlichen an Krebs-Nachsorgesprechstunden bzw. -zentren an universitären Tumorzentren (UCC) bzw. Comprehensive Cancer Centers (CCC) mit lokaler Vernetzung im ambulanten Bereich. So könnte gewährleistet werden, dass möglichst viele Nachsorge-Untersuchungen „zuhause“ und wenige Nachsorge-Untersuchungen durch das SpezialistenTeam im Zentrum durchgeführt werden.

Für die Versorgungsforschung ist es enorm wichtig, zu wissen, wie es den Patienten ergangen ist und wie es ihnen heute geht. Damit der Erfolg einer Behandlung umfassend bewertet werden kann, sollten Ärzte, Krankenkassen und Politik für diese neuen Strategien und Ansätze offen sein. Dann hat der Patient eine Chance, einen bislang oft vergeblich gesuchten spezialisierten Ansprechpartner in der Nachsorge zu finden.

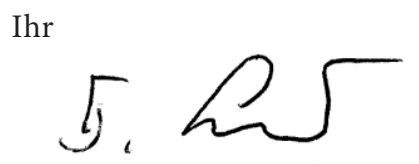

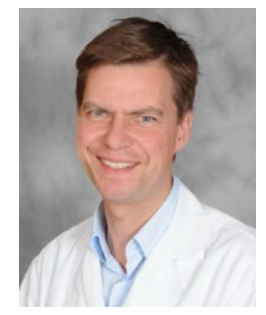

Prof. Dr. med. Thorsten Langer, MHBA Abteilung für Hämatologie, Onkologie und Zelltherapie,

Kinder- und Jugendklinik des Universitätsklinikums Erlangen

E-Mail: Thorsten.Langer@ uk-erlangen.de 